Memorias del VII Encuentro Nacional de Experiencias en la Enseñanza de la Biología y la Educación Ambiental y II Congreso Nacional de Investigación en la Enseñanza de la Biología

\title{
HALLAZGOS INICIALES SOBRE EL ESTADO DEL ARTE DE LA APROPIACIÓN SOCIAL DEL CONOCIMIENTO
}

\section{INITIAL FINDINGS ABOUT THE SOCIAL APPROPRIATION OF KNOWLEDGE}

\section{Gloria Escobar ${ }^{1}$ \\ Mónica García ${ }^{2}$}

Resumen (217/250):

Este escrito se hace como producto de un proyecto que se está adelantando en el momento, denominado ¿Cómo la Escuela se puede convertir en un vehículo para la Apropiación Social del Conocimiento?. Uno de los propósitos del trabajo es resaltar las bondades del ejercicio científico y tecnológico para el desarrollo sostenible de las sociedades, contribuyendo a una apropiación de la ciencia, la tecnología y la información en los ciudadanos que les permita utilizar el saber para estudiar, planear, anticiparse y proponer soluciones a los problemas presentes en su comunidad. En otras palabras, contribuir a que el individuo construya su propia capacidad de acción (Chaparro, 2000:19), con la confianza en sí mismo y en su país para generar nuevos conocimientos.

Para entender la importancia de trabajar en la Apropiación Social del Conocimiento (en adelante ASC) se hace pertinente saber en qué consiste, qué se ha hecho hasta el momento y cuál ha sido el papel de la escuela en este sentido. Esta ruta de trabajo tiene una serie de antecedentes y procesos evaluativos a nivel latinoamericano y nacional que muestran la pertinencia de trabajar alrededor del tema, y a la vez la necesidad de reestructurar la manera como se implementa, a través de programas específicos que buscan concretar sus objetivos. Algunos de estos aspectos son los que se presentarán a continuación.

\section{Abstract}

This is written as a product of a project that is advancing at the time, called How School can become a vehicle for the Social Appropriation of Knowledge? One purpose of the work is to emphasize the benefits of the science and technology exercise for sustainable development of societies, contributing to the

\footnotetext{
${ }^{1}$ Escuela Pedagógica Experimental. gloria.escobar@epe.edu.co

${ }^{2}$ Escuela Pedagógica Experimental. monica.garcia@epe.edu.co
} 
Bio-grafia Escritos sobre la Biologia y su Enseñanza.

Edición Extra-Ordinaria. ISSN 2027-1034 P.p $954-964$

Memorias del VII Encuentro Nacional de Experiencias en la Enseñanza de la

Biología y la Educación Ambiental y II Congreso Nacional de Investigación en la Enseñanza de la Biología

appropriation of science, technology and information in the public to allow them to use knowledge to study, plan, anticipate and propose solutions to the problems present in their community. In other words, to help the person to build his own action capacity (Chaparro, 2000:19), having self-confidence and trusting in his country to generate new knowledge.

In order to understand the importance of working on Social Appropriation of Knowledge (hereafter SAK) is relevant to know what it is, what has been done so far and what has been the role of the school in this regard. This work route has a number of antecedents and evaluative processes at national and Latin American level, which shown the pertinence of working around the topic and the need to restructure the way that is implemented through specific programs to achieve their goals. Some of these aspects are presented below.

Palabras Clave: Divulgación, Comunicación, Popularización y Apropiación Social del Conocimiento

Keywords: Dissemination, Communication, Popularization and Social Appropriation of Knowledge.

\section{Introducción}

Tanto a nivel nacional como internacional se ha planteado la importancia de promover una apropiación social del conocimiento científico y tecnológico al interior de las sociedades; para generar procesos dinámicos y continuos de aprendizaje social (Chaparro, 2001). En otras palabras, lo que se ha buscado es desarrollar capacidades y habilidades en las personas y en las organizaciones que les permitan enfrentar los desafíos de su entorno, crear alternativas de solución y proyectarse en oportunidades de desarrollo.

En estudios recientes que se han realizado en Bogotá se ha encontrado que la apropiación social de la ciencia y la tecnología en los ciudadanos, depende de los niveles educativos y los medios para acceder a la información, es decir, del estrato social al que pertenece cada persona (Daza en Observatorio Colombiano de Ciencia y Tecnología, 2009). Por lo cual, las escuelas se convierten en un vehículo esencial para la apropiación teniendo en cuenta que al menos éste primer nivel académico es asegurado para toda la población a nivel nacional sin importar su estrato. 
Bio-grafia Escritos sobre la Biologia y su Enseñanza.

Edición Extra-Ordinaria. ISSN 2027-1034 P.p $954-964$

Memorias del VII Encuentro Nacional de Experiencias en la Enseñanza de la

Biología y la Educación Ambiental y II Congreso Nacional de Investigación en la Enseñanza de la Biología

Pero, ¿Qué se entiende por Apropiación Social del Conocimiento Científico Y Tecnológico?

La articulación entre el conocimiento científico, tecnológico y la sociedad en general ha sido un tema que ha convocado la preocupación de un gran número de personas, organizaciones e instituciones tanto a nivel nacional e internacional, por su importancia para generar estrategias de desarrollo en los países, en relación al avance de la Ciencia, la Tecnología y la Información. Dicha articulación ha recibido diferentes denominaciones dependiendo de la comunidad que orienta las estrategias, aun cuando todas ellas comparten la pretensión de estimular la creación y consolidación de espacios para la comprensión, reflexión y debate de soluciones a problemas sociales, políticos, culturales y económicos en los cuales la generación y uso de conocimiento científico y tecnológico juegan un papel preponderante (Lozano y Maldonado, 2010:1). En la literatura la podemos encontrar como: Apropiación Social del Conocimiento "ASC", Apropiación Social del Conocimiento, la Tecnología y la Innovación "ASCTI", Comunicación de la Ciencia o Comunicación Pública de la Ciencia y la Tecnología, y/o Popularización de la Ciencia y la Tecnología.

El término "Popularización de la Ciencia y la Tecnología", ha sido ampliamente usado en Latinoamérica y consolidado a partir de numerosos encuentros multiregionales en los que se han expuestos las políticas y estrategias de diferentes países para propiciar la ASC. Según Mónica Lozano, se ha usado este término por dos consideraciones fundamentalmente: por un lado, es uno de los términos de más amplia utilización en la región (la creación de la Red de la Popularización de la Ciencia y la Tecnología para América Latina y el Caribe, Red Pop, en 1990 contribuyó a unificar el concepto), y por otro, porque es el concepto utilizado en los proyectos y programas realizados por el Convenio Andrés Bello desde la década de los ochenta (Lozano, 2005:19). Así mismo Julián Betancourt Mellizo menciona su preferencia por utilizar el término "popularización", en lugar de usar "comunicación" o "divulgación" de la ciencia debido esencialmente a:

1. Existen diversas formas de comunicar el conocimiento científico y tecnológico; y la popularización es solo un tipo más de comunicación. También existen otras formas de comunicar, como: la comunicación intracientífica, intercientífica y pedagógica.

2. La divulgación aunque comparte con la popularización la idea de estar dirigida a un amplio auditorio, solo se dedica a informar sobre algún tema sin necesidad de buscar una comprensión y aplicación del mismo para tomar decisiones. 
Bio-grafia Escritos sobre la Biologia y su Enseñanza.

Edición Extra-Ordinaria. ISSN 2027-1034 P.p $954-964$

Memorias del VII Encuentro Nacional de Experiencias en la Enseñanza de la

Biología y la Educación Ambiental y II Congreso Nacional de Investigación en la Enseñanza de la Biología

Con base en esta información se podría decir que las enciclopedias, las conferencias y las exposiciones convencionales en los museos han sido estrategias de divulgación de la ciencia más que de popularización de la misma.

En cambio, para Luis Estrada (en Lozano, 2005) la distinción debe ser entre: difusión, divulgación y comunicación de la ciencia. Entendiendo por difusión, la propagación del conocimiento entre especialistas; por divulgación, las actividades dirigidas a presentar la ciencia al público en general; y por comunicación, el intercambio de saberes y experiencias a través del diálogo entre personas.

En nuestra investigación se opta por utilizar el término de Apropiación Social del Conocimiento, la Tecnología y la Innovación, y no el de "popularización", debido a dos razones. Primero, porque en concordancia con Sandra Daza (en Observatorio Colombiano de Ciencia y Tecnología, 2009:67) la apropiación significa "hacer algo suyo", expresión que involucra encontrar que el conocimiento es útil para las personas en la toma de decisiones sobre su vida diaria, es decir implica una participación directa en la generación de conocimiento. Y, Segundo, porque la política científica y tecnológica promovida por el país toma esta aserción para justificar sus estrategias a nivel nacional para lograr una mejor apropiación social del conocimiento científico y tecnológico. La idea no es negar la historia que tiene el término popularización de la ciencia sino plantear el uso de "apropiación social" como término adecuado para la búsqueda concreta de una cultura científica.

\section{Metodología}

El presente trabajo se ha llevado a cabo a través de la revisión de materiales escritos desde un enfoque cualitativo interpretativo en la perspectiva de análisis documental propuesta por Araceli de Tezanos (2004) en su libro "Una etnografía de la etnografía, aproximaciones metodológicas para la enseñanza del enfoque cualitativo-interpretativo para la investigación social". Los materiales escritos que han sido recopilados incluyen un amplio espectro que va desde la literatura científica de referencia hasta decretos, actas de sesiones, trabajos de grado, memorias de eventos, pasando por artículos de revistas de tipo magazine, de circulación amplia y general. La revisión bibliográfica que se ha hecho hasta el momento ha sido esencial para el diseño de la investigación en torno a la pregunta ¿Cómo la escuela se puede convertir en un vehículo para la Apropiación Social del Conocimiento?

En este acercamiento al estado del arte, se ha intentado construir un tejido "que entrelaza las diferentes posturas y las semejanzas y divergencias entre las mismas, formuladas por una variedad de autores (De tezanos, 2004). La intención 
Bio-grafia Escritos sobre la Biologia y su Enseñanza.

Edición Extra-Ordinaria. ISSN 2027-1034 P.p $954-964$

Memorias del VII Encuentro Nacional de Experiencias en la Enseñanza de la

Biología y la Educación Ambiental y 11 Congreso Nacional de Investigación en la Enseñanza de la Biología

de ésta primera fase de revisión documental es construir la bases para la interpretación de la información que resulte de la investigación en la fase posterior de "construcción de datos" (según Dobles et al., 1996).

En palabras de Schwab (en Kemmis, 1993), lo importante es animar a los educadores a hacer interpretaciones de las circunstancias sociales y educativas actuales en situaciones reales concretas y a tomar decisiones sobre la base de su razonamiento, en este caso, alimentado por un movimiento dialéctico entre la teoría y la práctica.

\section{Resultados y discusión}

Investigaciones recientes realizadas en nombre de la "popularización de la ciencia", han encontrado dos grandes modelos de apropiación social del conocimiento: el de déficit y el democrático (Durant en Lozano, 2005). Mientras para el modelo de déficit el público carece de conocimientos científicos y la labor de la popularización es suplir estas carencias (Lozano, 2005:62), para el modelo democrático el público es reconocido como poseedor de conocimientos y experticia, además de valores e intereses que son útiles en la reflexión sobre la aplicación de la ciencia en contextos sociales específicos (Lozano, 2005:62); por lo que éste último se refiere a procesos de comunicación que van en doble vía entre ciencia y el público.

\section{¿Cuál es la situación en América Latina?}

En América Latina aunque durante varios años se trabajó con propuestas desde el modelo de déficit, adoptando modelos de desarrollo externos impulsados por políticas internacionales con formas de participación pública limitadas; en la actualidad, ha surgido una nueva tendencia por adoptar estrategias de apropiación social de la ciencia ligadas a un modelo democrático, que busca encontrar salidas de acuerdo con las características propias de los contextos científicos, sociales, económicos y políticos de la región. Algunas de las estrategias que se han utilizado en el contexto latinoamericano para proyectar un modelo democrático para la Apropiación Social del Conocimiento Científico y Tecnológico en las distintas regiones son: 
Bio-grafia Escritos sobre la Biologia y su Enseñanza.

Edición Extra-Ordinaria. ISSN 2027-1034 P.p $954-964$

Memorias del VII Encuentro Nacional de Experiencias en la Enseñanza de la Biología y la Educación Ambiental y II Congreso Nacional de Investigación en la Enseñanza de la Biología

a) Red de Popularización de la Ciencia y de la Tecnología en América Latina y del Caribe o Red-POP (http://www.redpop.org).

b) Foro Iberoamericano de Comunicación y Divulgación Científica (organizado por la Red Territorial EXPLORA, en la región del Bío Bío de Chile).

c) Jornadas Iberoamericanas de evaluación de la Comunicación de la Ciencia (apoyadas por la Organización de Estados Americanos (OEA) y la Agencia Española de Cooperación Internacional (AECl).

d) Congreso Iberoamericano de Ciudadanía y Políticas Públicas en Ciencia y Tecnología (encuentro organizado por la Fundación Española de Ciencia y Tecnología (FECYT), la OEl y el Centro REDES).

e) La declaración de Toronto (aunque es una estrategia que va más allá del contexto latinoamericano, se constituye en una base legal a nivel mundial que promueve la cooperación entre los países).

Estos encuentros se podrían tomar como parte de un modelo democrático debido a que van más allá de la difusión y de la divulgación; además, plantean una concepción de conocimiento que supera la imagen de éste como un campo certero y seguro, con poca posibilidad de cuestionamiento. Lo que buscan es plantear la necesidad de inducir procesos en los que el público participe políticamente en la toma de decisiones en ciencia y tecnología en igualdad de condiciones con los científicos (Lozano, 2005), desde una mirada más profunda su imagen de conocimiento está más relacionada con la parcialidad y provisionalidad, que con la certeza y la invariabilidad.

\section{¿Cuál es la situación en Colombia?}

Aunque desde el año de 1990, con La ley 29 expedida en octubre de ese mismo año, en el congreso de la república se establecieron algunos artículos reglamentarios para el fomento de la investigación científica, el desarrollo tecnológico y para explicitar las facultades extraordinarias concedidas al Fondo Colombiano de Investigaciones Científicas y proyectos especiales "Francisco José de Caldas" (Colciencias); solo hasta el año 2009, con la ley 1286 de enero 23 de 2009, se consolidó realmente una política dirigida no solo a transformar a Colciencias en departamento administrativo sino a ampliar los campos de acción en torno a la apropiación social del conocimiento, la tecnología y la información. 
Bio-grafia Escritos sobre la Biologia y su Enseñanza.

Edición Extra-Ordinaria. ISSN 2027-1034 P.p $954-964$

Memorias del VII Encuentro Nacional de Experiencias en la Enseñanza de la

Biología y la Educación Ambiental y II Congreso Nacional de Investigación en la Enseñanza de la Biología

Algunas de las estrategias y mecanismos a través de los cuales el actual Centro Administrativo en Ciencia, Tecnología e Información Colciencias ha intentado aportar en los procesos de Apropiación de la Ciencia, la Tecnología y la Información son:

a) A través de la Semana Nacional de la Ciencia, la tecnología y la Información (realizada desde el 2006 por Colciencias).

b) A través de la construcción de un centro de recursos para la apropiación (orientado por Colciencias y Maloka) que realiza un rastreo de las experiencias elaboradas por: empresas, centros de investigación, universidades, ONGs, etc.

c) A través de la realización de foros de discusión (Hasta el momento se han realizado dos: sobre Biocombustibles (2008) y sobre Política Forestal (2010)).

d) A través de aulas rodantes (que inició en el año 2009 en conmemoración al año internacional de la astronomía, y a partir del cual se diseñó y adecuó un Observatorio Astronómico Itinerante)

e) A través del foro de la ASCTI (por ejemplo, en Octubre de 2010 se desarrolló un Foro Internacional en EAFIT donde se socializó la estrategia de ASCTI y se puso a discusión con expertos internacionales.

f) A través de la construcción de la Estrategia de Apropiación Social del Conocimiento, la Tecnología y la Información ASCTI.

De todas las estrategias mencionadas anteriormente es importante profundizar en el último aspecto, pues la Estrategia de Apropiación Social del Conocimiento, la Tecnología y la Información ASCTI escrita por Colciencias, es la base de las demás propuestas a nivel nacional. Según ésta, la ASC es el fundamento de cualquier forma de innovación porque el conocimiento es una construcción compleja que involucra la interacción de distintos grupos sociales (Colciencias, 2010); en este sentido, el conocimiento no solo se ve como el resultado de la interacción entre seres diversos sino como una posibilidad de acción de los sujetos, por ejemplo para la solución de los problemas que hacen parte de su entorno. 
Bio-grafia Escritos sobre la Biologia y su Enseñanza.

Edición Extra-Ordinaria. ISSN 2027-1034 P.p $954-964$

Memorias del VII Encuentro Nacional de Experiencias en la Enseñanza de la

Biología y la Educación Ambiental y II Congreso Nacional de Investigación en la Enseñanza de la Biología

\section{¿Cuál es la situación en las escuelas?}

La intención de la investigación que se está adelantando se centra en el estudio de estrategias que se desarrollan a nivel escolar para lograr la ASC, por ello es pertinente el inventario que realizan Manjarrés y otros (2010:18) sobre los programas que en Colombia promueven la ASCTyl, estos son Ondas (Colciencias), Estudiantes como Científicos (Parque Explora), Pequeños Científicos (Universidad de los Andes), Expociencia Infantil, Juvenil y Universitaria (Asociación Colombiana para el Avance de la Ciencia [ACAC]), los Museos de la Ciencia y el Juego (Universidad Nacional), los jardines botánicos, los museos interactivos como Maloka, los clubes de ciencia y tecnología y las organizaciones juveniles orientadas a la formación científica temprana como Buinaima, la Fundación FISS, la Central Científica, la Federación Internacional de Sociedades Científicas y la Fundación Escuelas Libres de Investigación Científica para Niños (ELIC).

A continuación se presentan algunos de los resultados de los estudios que han realizado: Mónica Lozano (2008) sobre "El estado de la evaluación de las actividades científicas infantiles y juveniles: una panorámica regional”; y Rátiva y otros (2011) sobre la evaluación de las "Actividades de apropiación social de la ciencia y la tecnología y los espacios de encuentro con los públicos en Colombia: una mirada a los proyectos apoyados por Colciencias 2005-2010".

I. El trabajo que Colombia desarrolla en el campo de las ferias de la ciencia se resalta por: el evento Feria Expociencia -Expotecnología y las conferencias del Encuentro con el futuro (organizados por la Asociación Colombiana para el Avance de la Ciencia y la Tecnología-ACAC), los clubes de ciencias de la ACAC y Maloka, y el programa Ondas de Colciencias. En cuanto al ítem de concursos y olimpiadas, no hay reporte de un evento que se realice en Colombia con estas características.

II. En la sistematización de 102 proyectos que se ejecutaron entre los años 2005-2010 con el apoyo de Colciencias, se encontró que en un $72 \%$ se impulsa el espacio de comunicación científica, un $25 \%$ el espacio de producción y difusión del conocimiento, y un 3\% al espacio de difusión de la política en CyT.

III. La comunicación científica que usan los medios de comunicación masiva (radio, prensa, televisión, internet), las exposiciones y museos, obras de teatro, ferias de CyT, colecciones editoriales y actividades de periodismo científico; se caracterizan por una comunicación vertical, unidireccional y masiva, dando mayor importancia al proceso de transmisión de información 
Bio-grafia Escritos sobre la Biologia y su Enseñanza.

Edición Extra-Ordinaria. ISSN 2027-1034 P.p $954-964$

Memorias del VII Encuentro Nacional de Experiencias en la Enseñanza de la

Biología y la Educación Ambiental y II Congreso Nacional de Investigación en la Enseñanza de la Biología

sobre ciencia y tecnología, a unos públicos que en la mayoría de los casos corresponde a la franja infantil y juvenil. Rátiva y otros (2011:174) agregan que esta forma de actuar hace recordar el "modelo de déficit" de Lewenstain en el año 2003, donde las audiencias se consideran como una especie de "recipiente vacio".

IV. Se resalta la débil continuidad en el tiempo de las propuestas (producciones televisivas y periodismo científico), que además se encuentran principalmente dirigidas a un público en general; elemento que evidencia la indeterminación y en muchos casos la poca claridad de a quiénes se quiere interpelar directamente.

V. Los recursos destinados para la ASC son pocos por lo que es necesario evaluar y hacer seguimientos para privilegiar iniciativas que le apunten a la calidad de los contenidos garantizando procesos de más largo aliento.

VI. El apoyo financiero ha estado dividido en dos campos: a) la producción de materiales impresos (libros y revistas) y b) la realización de eventos. Dentro de las entidades que impulsan estas iniciativas están las instituciones de educación superior $(51,8 \%)$, ONG'S, asociaciones, agremiaciones profesionales y entidades mixtas $(26,8 \%)$, centros de investigación y desarrollo, las empresas, las entidades del gobierno central y las instituciones privadas sin fines de lucro. Maloka es la entidad con mayor índice de participación (ACAC, 13,3\%, Maloka 40\%).

Así, teniendo en cuenta los aspectos anteriormente mencionados se podría decir que aunque han existido varias estrategias para involucrar a las comunidades escolares en las dinámicas de ASC, ha faltado iniciativa por parte de los mismos establecimientos educativos para proponer alternativas de trabajo que promuevan al menos el debate y sino la participación en el sentido que aquí se plantea, para superar las relaciones verticales entre los expertos y los públicos en general, y llegar a relaciones más horizontales y procesos más democráticos.

\section{Conclusiones}

En la revisión documental realizada hasta el momento se observa que existe una intención decidida para estructurar una política sobre la ASC a nivel nacional y latinoamericano. A partir de estas políticas se han diseñado e implementado programas que logren cumplir con los propósitos que se han planteado en las diferentes políticas. Además, es interesante ver que los mismos impulsores de dichas políticas y programas están en actitud de revisar permanentemente, a través de eventos y de documentos, la incidencia de estas políticas y programas. 
Bio-grafia Escritos sobre la Biologia y su Enseñanza.

Edición Extra-Ordinaria. ISSN 2027-1034 P.p $954-964$

Memorias del VII Encuentro Nacional de Experiencias en la Enseñanza de la

Biología y la Educación Ambiental y 11 Congreso Nacional de Investigación en la Enseñanza de la Biología

La Asociación Colombiana para el Avance de la Ciencia y la Tecnología (A.C.A.C.), Maloka y Colciencias son las instituciones que se identifican como las abanderadas en el proceso de la ASCCyT en nuestro país a nivel escolar. Sin embargo hasta el momento no se hay evidencias donde se observe que se desarrolla como un proceso sistemático que se implemente en todas las instituciones escolares de nuestro país, sino que más bien en estas actividades participan las instituciones escolares que muestran interés en este campo formativo. Además se podría pensar que en las instituciones donde se desarrollan estas actividades no hacen parte de un proceso continuo sino que responde a situaciones coyunturales.

Dentro de la diversidad de programas caracterizados en Colombia se encuentra que éstos se han estructurados a partir de entidades como Colciencias y ACAC para que los colegios, y en general la población escolar, acceda a sus programas, sin embargo no se identifican programas que se hayan estructurado desde la escuela misma o que de manera sistemática se desarrolle en estas instituciones formativas. Este aspecto es el que nos llevaría a exaltar la pertinencia de nuestra propuesta investigativa.

\section{Bibliografía}

CHAPARRO, Fernando (2000). Conocimiento, Aprendizaje y Capital Social como Motor de Desarrollo. Rev. Ciencia da Informacao, Jan/Apr, 2001, vol. 30. No 1, p19-31, Brasil.

COLCIENCIAS (2005). La percepción que tienen los colombianos sobre la ciencia y la tecnología. Bogotá (Colombia): Panamericana formas e impresos S.A.

DE TEZANOS, Araceli (2004). Una etnografía de la etnografía, aproximaciones metodológicas para la enseñanza del enfoque cualitativo-interpretativo para la investigación social. Santafé de Bogotá, D.C.: Ediciones Antropos, Colección Pedagógica S. XXI.

DOBLES YZAQUIRRE, María Cecilia; ZUÑIGA CÉSPEDES, Magaly; GARCIA FALLAS, Jackeline (1996). Investigación en Educación: procesos, interacciones, construcciones. San José, Costa Rica: Editorial Universidad Estatal a distancia, EUNED.

KEMMIS, Stephen (1993). El currículum: más allá de la teoría de la reproducción. Madrid (España): Ediciones Morata S.L. 
Bio-grafia Escritos sobre la Biologia y su Enseñanza.

Edición Extra-Ordinaria. ISSN 2027-1034 P.p $954-964$

Memorias del VII Encuentro Nacional de Experiencias en la Enseñanza de la Biología y la Educación Ambiental y II Congreso Nacional de Investigación en la Enseñanza de la Biología

LOZANO, Mónica, y SANCHEZ MORA, Carmen (2006). Evaluando la Comunicación de la ciencia: una perspectiva latinoamericana. Memorias de las Jornadas Iberoamericanas sobre Criterios de Evaluación de la Comunicación de la Ciencia, realizadas en Cartagena de Indias, Colombia, del 14 al 17 de Noviembre de 2006. http://ocyt.org.co/html/archivosProyectos/Capitulo Daza.pdf

LOZANO, Mónica (2005). Programas y experiencias en popularización de la ciencia y la tecnología, panorámica desde los países del Convenio Andrés Bello. Bogotá (Colombia): Convenio Andrés Bello.

OBSERVATORIO COLOMBIANO DE CIENCIA Y TECNOLOGÍA (2009). Percepciones sobre la ciencia y la tecnología en Bogotá. Bogotá (Colombia): Observatorio Colombiano de Ciencia y Tecnología.

OEI (2008). Declaración de Toronto: $5^{\circ}$ Congreso Mundial de Centros de Ciencias. 28 de julio de 2008. http://www.oei.es/noticias/spip.php?article3113

MANJARRÉS, María Elena, MEJÍA Marco Raúl, CIPRIANO SASTRE Jenny y Martínez Andrea (2010). Las Ferias Infantiles y Juveniles de Ciencia, Tecnología e Innovación como Espacios de Formación y Apropiación Social. Lineamientos pedagógicos del Programa Ondas.

http://www.alcaravan.org.co/ondas/registros sis/Manual Ferias final.pdf

RÁTIVA, M. Nathali J., LOZANO Borda, Marcela y MALDONADO Oscar (2010). Actividades de apropiación social de la ciencia y la tecnología y los espacios de encuentro con los públicos en Colombia, una mirada a los proyectos apoyados por Colciencias 2005 - 2010. Folios 25, 2011, Facultad de Comunicaciones, Universidad de Antioquia http://aprendeenlinea.udea.edu.co/revistas/index.php/folios/article/viewFile/10606/ $\underline{9729}$

Sin Autor (2010). Ciencia, tecnología e innovación deben ser democráticas. Artículo tomado de http://www.eafit.edu.co/foro-taller-ascti/novedades.html 